\title{
DESARROLLO DE COMPETENCIAS \\ EN LAS ÁREAS DE TECNOLOGÍA Y MATEMÁTICAS \\ A TRAVÉS DE MARCOS CONCEPTUALES
}

\author{
Luis Facundo Maldonado G. Ph.D. \\ Omar López Vargas M. Sc. \\ Jaime Ibáñez lbáñez M. Sc. \\ Héctor Rojas Sarmiento \\ Luis Carlos Sarmiento*
}

\begin{abstract}
This paper summarizes an educational innovation developed with sixth grade students in mathematics and technology education. It uses a shell to produce hypertexts together with a knowledge representation schema.

When this instructional strategy is introduced, classrooms can be changed to intellectual production spaces. The teacher role is focused on monitoring student earning processes, environmental support, and advice on conceptual, methodological and technical tasks. Students are very oriented to build knowledge representations which summit for validation, first of a/l to their partners, next to their teachers, and finally to their academic community

Designing hypertexts based on a frame system consistently help the students to improve cognitive, metacognitive, colaborative and motor skills. This way of designing computer supported education environments, which uses know/edge representation schemas consistent with scientific domains, is a constructivistic approach positively related to meaningful learning.
\end{abstract}

\section{RESUMEN}

Este trabajo presenta una síntesis de una innovación educativa desarrollada con estudiantes del Centro educativo Distrital Venecia de grado sexto de educación básica en las áreas de matemática y tecnología. Utilizó un ambiente para elaborar hipertextos y un sistema estructurado de representación de conocimiento en su concepción y desarrollo.

Con esta estrategia metodológica se transforma el aula de clase en un espacio de producción intelectual. El rol del profesor se centra en el monitoreo de procesos, mantenimiento del ambiente y asesoría sobre dimensiones conceptuales, metodológicas y técnicas. El estudiante se orienta decididamente a la construcción de representaciones que somete a validación de sus pares, de sus profesores y de la comunidad académica en la cual se desempeña.

Los resultados muestran que el diseño de hipertextos a través de la estructura de Sistema de Marcos desarrolla en el estudiante habilidades cognitivas, metacognitivas, colaboratívas y motrices. Esta forma de utilizar ambientes basados en computador diseñados con base en sistemas de representación consistentes con el desarrollo de la

* Profesores Universidad Pedagógica Nacional 
disciplina científica es una aproximación constructiva que da como resultado aprendizaje significativo.

Palabras claves: Desarrollo de competencias, aprendizaje significativo, representación de conocimiento, metacognición, marcos conceptuales, aprendizaje mediante colaboración, software educativo, aprendizaje de matemática, aprendizaje de tecnología, educación matemática, educación en tecnología.

\section{INTRODUCCIÓN}

La alianza entre el Centro Educativo Distrital Venecia y el grupo TECN CE de a Universidad Pedagógica Nacional que dio origen a la innovación educativa que aquí relatamos, surgió de un anhelo común: crear ambientes de aprendizaje basados en computador para desarrollar competencias cognitivas al tiempo que autonomía y colaboración en el proceso de aprendizaje en niños de educación básica.

El grupo TECNICE ha venido comprometido de manera sostenida en el desarrollo de proyectos orientados a entender los mecanismos y procesos relacionados con tres tópicos centrales en este proyecto: La autonomía, la colaboración y la representación de conocimiento en el aprendizaje. Los escenarios para su estudio privilegian el uso de computadores en la perspectiva de la pedagogía computacional.

En una innovación previa (Ortega et Al., 2001) se validó un modelo que integró los siguientes elementos: un ambiente de tarea compuesto por problemas centrales y fuentes de información, especialmente en formato de texto; trabajo individual, donde el estudiante identificaba el problema, formulaba sus juicios de metamemoria y hacia sus búsquedas de solución: un ambiente de trabajo colaborativo de contrastación y ajuste de solución; un ambiente para la autoevaluación y finalmente un ambiente de acreditación de logros de aprendizaje. En el desarrollo de esa experiencia participaron dos cursos de grado sexto de educación básica, uno de niños en jornada de la mañana y otro de adultos en jornada nocturna en las áreas de matemática, español, ciencias y tecnología.

Los estudiantes inicialmente mostraron alta dependencia de las orientaciones del profesor. Frecuentemente tuvieron dificultades tanto en el trabajo individual como colaborativo. El aprendizaje de contenidos fue también lento en sus inicios. Después del primer mes de trabajo se hizo evidente la consolidación de la capacidad para definir metas, cumplir agendas y hacer búsquedas de información. A partir del tercer mes los estudiantes avanzaron mas rápido en el aprendizaje de contenidos que los otros cursos del mismo colegio e invirtieron más tiempo en el estudio de las asignaturas. Al terminar el primer semestre y en el siguiente obtuvieron un rendimiento superior en todas las áreas de estudio en comparación con los demás cursos del mismo grado.

La estructura de marcos, también denominada red semántica estructurada (Sowa, 1987) sirvió de base para un estudio en el cual se comparó la formación de conceptos en un grupo estudiantes que utilizaba un software para elaborar hipertextos con otro que estudiaba hipertextos ya elaborados por expertos (Maldonado, Ortega, Sanabria y Macías, 2002). Este trabajo generó la estructura de software que se utiliza en esta innovación. 
El proyecto que aquí presentamos fue financiado por El Instituto para el Desarrollo Pedagógico y la Investigación Educativa IDEP. Se implementó y ejecuto en el CED Venecia durante el año 2001, en el curso 604, teniendo como asesor al grupo de investigación TECNICE de la Universidad Pedagógica Nacional. La innovación cuenta con la pagina Web www.cedvene a.cb.net, a la cual se pueden remitir los interesados.

\section{REFERENTES TEÓRICOS}

El proceso de innovación pedagógica se orienta al desarrollo de competencias básicas a través de la estructura de marcos, articulado con la elaboración de una pieza de software - hipertexto- en La cual se refleja la representación de conocimiento de la unidad temática en estudio.

\section{- Sistema de Marcos}

Un marco es una estructura de atributos, normalmente llamados ranuras, con valores asociados que expresan un concepto temático. Un sistema de marcos se forma conectando varios marcos, teniendo en cuenta que el valor de un atributo de un marco puede ser a su vez otro marco. En la gráfica $\mathrm{N}^{0} 1$ se describen las partes que componen un sistema de marcos.

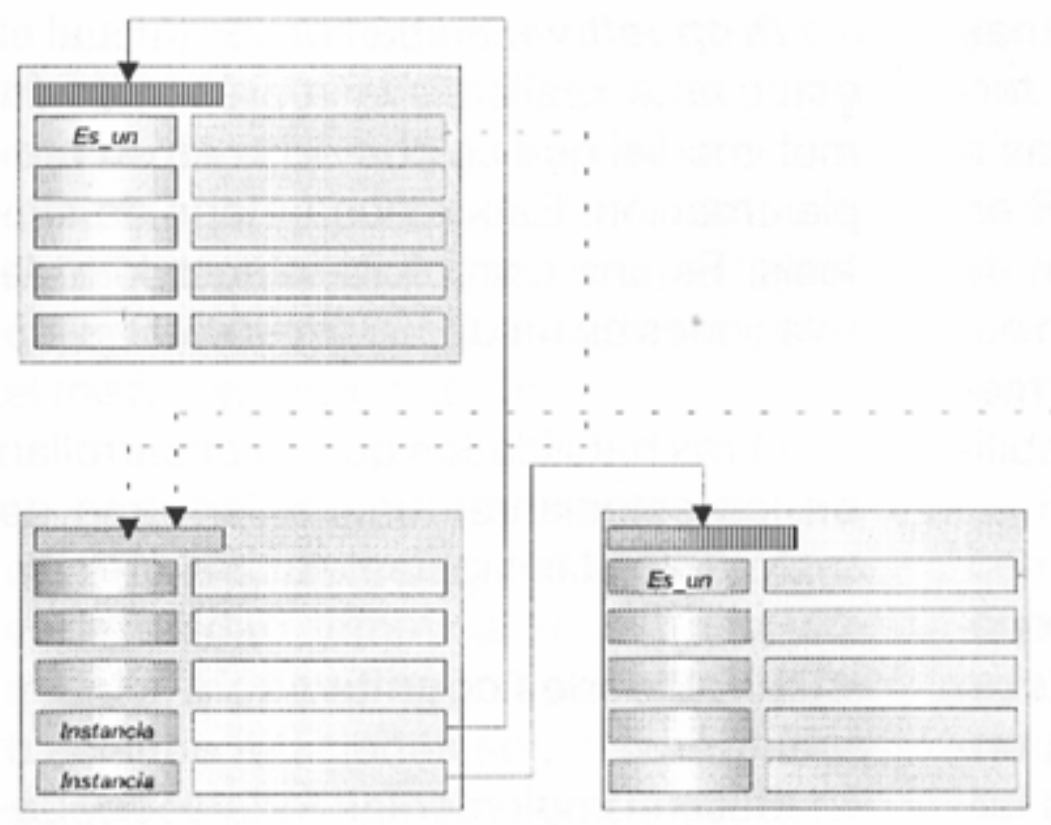

Convenciones:

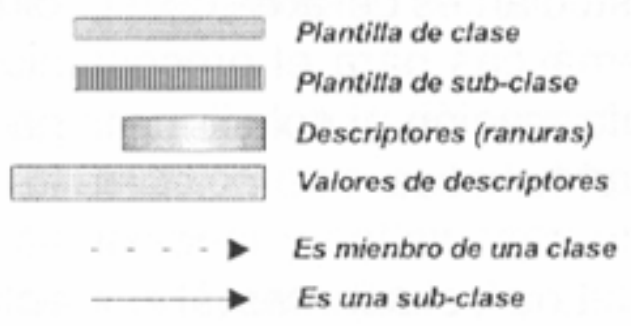

Gráfica 1. Componentes de un sistema de marcos. 
Si un marco representa una clase, su instancia representa un elemento de dicha clase. La relación es-un, y la relación instancia, son relaciones inversas que conectan subclases con clases y viceversa. Las sub-clases heredan los descriptores de la clase, cambiando los valores de los descriptores en la nueva plantilla.

Las plantillas pueden representarse en notación alternada de rectángulo y ranura. Los nombres asignados a los descriptores son los nombres de los enlaces que parten de ese nodo de la plantilla. El lenguaje de plantilla descriptor y valores de descriptores, resultan más estructurados y, por ende, más claros que el lenguaje de nodo y enlaces (redes semánticas).

\section{- Herencia en la estructura de marcos}

Es una relación entre clases y subclases (hijas) que hace que éstas tengan las propiedades de la clase superior. Una sub-clase hereda las propiedades paternas, pero, tiene, además atributos propios que le dan mayor especificidad. La clase superior tiene menos propiedades que la subclase y se aplica a más elementos, en tanto, La subclase tiene más propiedades — las de su padre y las propias - pero se aplica a menos casos.

Las relaciones de herencia generan estructuras arbóreas: las clases superiores se ramifica y lo mismo sucede con Las subclases. Los niveles inferiores muestran más ramas que los niveles superiores. El árbol de herencia constituye una forma de ordenamiento del conocimiento que tenemos de los sistemas.

\section{- Desarrollo de competencias a través de un sistema de marcos}

La metodología de sistemas de marcos, requiere de varias etapas para representar un conocimiento. Se inicia con la búsqueda de información para proceder Luego al diseño de esquemas que expresen claramente los conceptos, proseguir con el desarrollo del hipertexto, someterlo a prueba y corregirlo.

A nuestro entender, la representación de conocimiento por medio de un sistema de marcos se puede convertir en un método de aprendizaje que Llena varias de las expectativas actuales en la formación básica y que son expresadas a través de entidades como el ICFES en sus orientaciones sobre evaluación de competencias básicas. En efecto, un estudiante capacitado a través de esta metodología articula las siguientes habilidades:

Interpretativa: la actividad de búsqueda de información y de representación que desarrolla el alumno, lo lleva a encontrar significados en textos y otras formas de información disponibles.

Argumentativa: se crean condiciones para que el estudiante dé razón de la representación de conocimiento elaborada. El estudiante negocia con sus compañeros en condiciones de colaboración tanto el contenido como la forma de representación. Luego, con los docentes evalúa Los procesos argumentando posiciones y con la comunidad en Los procesos de socialización de los trabajos, explica la razón de ser tanto de la estructura del hipertexto como sus diferentes componentes. 
Propositiva: etapa final en la cual el estudiante realiza una representación multimedial que se convierte en su propia creación. Esta es una etapa de síntesis. Es una estructura integradora de relaciones en unidades significativas.

Otras habilidades que se desarrollan en los estudiantes bajo el sistema de marcos son las siguientes:

\section{- Habilidades cognitivas}

Buscan implementar, fortalecer y desarrollar habilidades cognitivas en los estudiantes del área de tecnología y matemáticas para el procesamiento de la información al solucionar una situación problemática.

Los estudiantes al enfrentarse a la representación de conocimiento, a través de sistemas de marcos, emplean estructuras y procesos para seleccionar, transformar, decodificar, almacenar, recuperar y generar información tendiente a la solución del problema planteado. El siguiente gráfico muestra cómo está estructurada esta competencia.

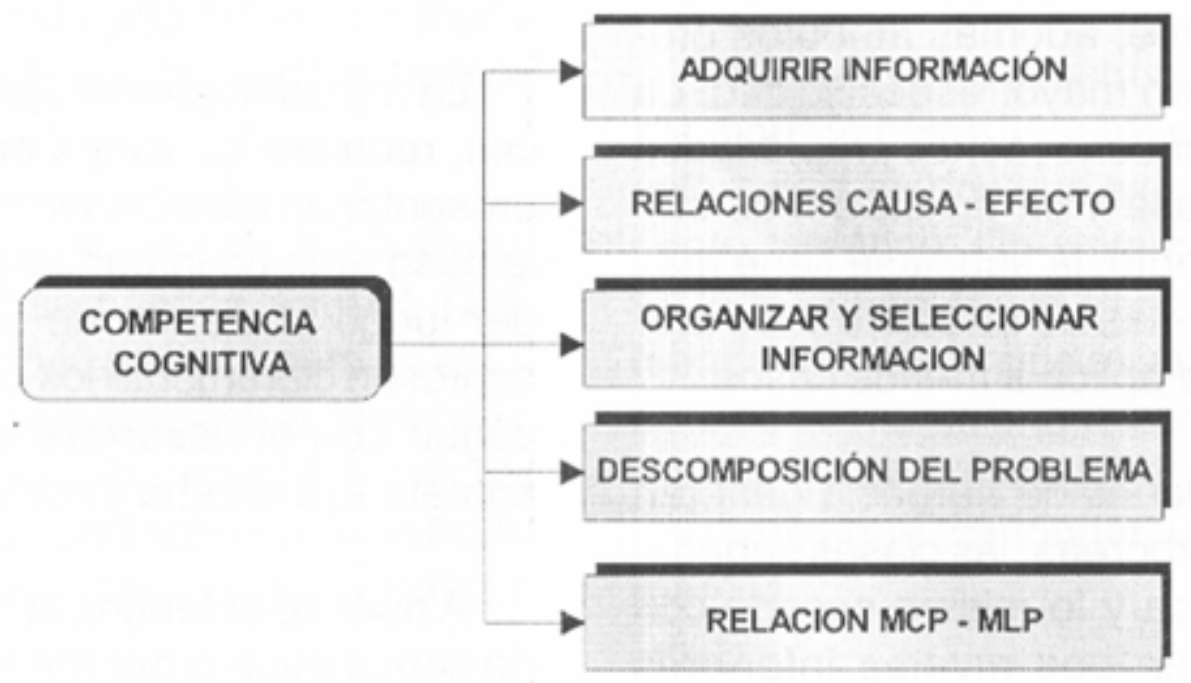

Gráfica 2. Habilidad cognitiva.

La representación de conocimiento a través de sistema de marcos, se inicia con la etapa de adquisición de información, la cual se puede encontrar disponible en diferentes fuentes como personas, objetos, videos, fotografías, textos, y en el mismo entorno natural.

El estudiante, en la medida en que maneje la información recolectada para cada unidad temática en estudio, comienza a operar cognitivamente, estableciendo relaciones según las ranuras seleccionadas entre el nombre de la ranura, sus respectivos contenidos y las definiciones de cada ranura.

En la organización de la información adquirida el estudiante se ve en la necesidad de seleccionar y definir cada una de las ranuras, así como las instancias y sus relaciones de herencia entre padres e hijos o, entre clases y subclases. Una vez, organizada la información determinan los contenidos de las ranuras. 
La habilidad cognitiva de descomposición de un problema en subproblemas, se pone en ejercicio cuando en una estructura de marcos, el objeto temático se descompone en nodos padre y en nodos hijos. Esta es una forma de subdividir el problema en varios momentos o etapas. Por ejemplo, el estudiante define como primer paso un nodo padre y posteriormente, cada uno de los nodos hijos y, posteriormente cada uno de estos pueden ser subdivididos en otros nodos hijos.

En este proceso los estudiantes hacen uso de sus conocimientos previos conservados en su memoria de largo plazo (MLP), la cual integran con la información que van adquiriendo en su memoria de trabajo o de corto plazo (MCP) para resolver cada situación.

\section{- Habilidad colaborativa}

Esta Habilidad se desarrolla en el trabajo en equipo. La representación de conocimiento, se consolida mediante negociación tomando como base el saber de cada uno de Los integrantes del grupo. De esta forma la habilidad colaborativa genera en los estudiantes la construcción y reconstrucción de conocimientos en las áreas de estudio. Una vez, que un grupo ha definido la representación de conocimiento definitiva, la plasma en un Hipertexto y en el siguiente paso la somete a validación por los otros equipos del curso.

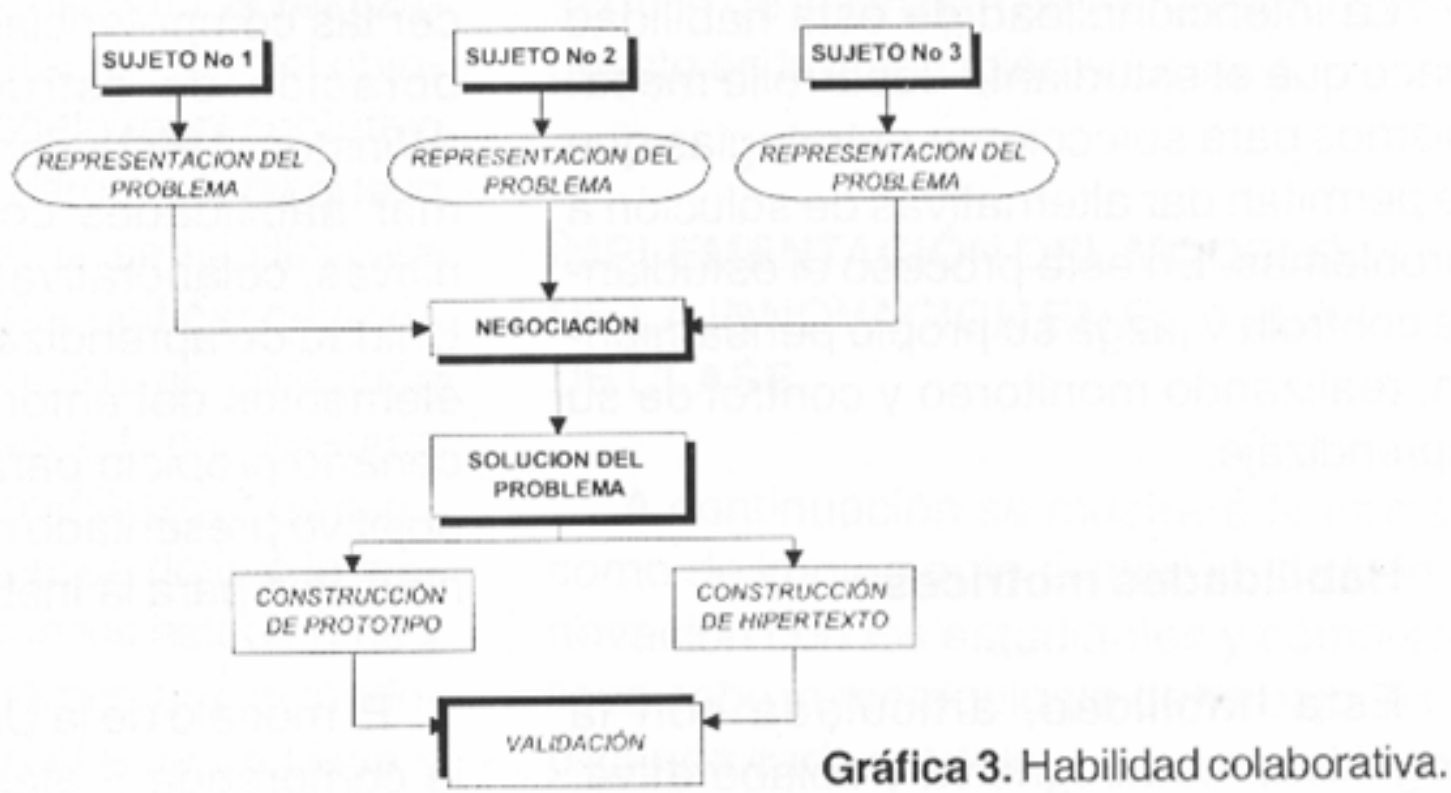

\section{- Habilidad metacognitiva}

Busca implementar, desarrollar y fortalecer en el estudiante la capacidad de conocimiento sobre su propio conocimiento. Las guías de trabajo contienen activadores de juicios de metamemoria que los llevan a valorar, bien sea el nivel de seguridad de su aprendizaje previo, la dificultad para enfrentar retos similares y la calidad del aprendizaje que se está adquiriendo. Estos activadores son preguntas que inducen a los estudiantes a elaborar juicios sobre sus procesos de aprendizaje en sus diferentes etapas. Los activadores se presentan a los estudiantes tanto a nivel individual como a nivel de grupo para de tal manera que haya juicios individuales y juicios construidos colaborativamente. Investigaciones previas han mostrado que la emisión de estos juicios da origen a fuerzas 
motivacionales que hacen que los estudiantes activen estrategias sistemáticas de solución de problemas. Los procesos reflexivos y de monitoreo del aprendizaje se activan para fortalecer la autonomía a nivel motivacional y de dirección del proceso de aprendizaje (Maldonado y Andrade, 2001 y Maldonado, 2001).

La intencionalidad de esta habilidad hace que el estudiante desarrolle mecanismos para seleccionar estrategias que le permitan dar alternativas de solución a problemas. En este proceso el estudiante controla y juzga su propio pensamiento, realizando monitoreo y control de su aprendizaje.

\section{- Habilidades motrices}

Esta habilidad, articulada con la cognitiva, metacognitiva y colaborativa, básicamente comprende las siguientes etapas:

MANIPULACIÓN: Comprende la adquisición de habilidades y destrezas motrices que le permiten a los estudiantes operar y manipular objetos e instrumentos para dar respuesta a los problemas. Esta habilidad se hace realidad cuando el estudiante interactúa con el computador al momento de construir el hipertexto y otros elementos como materiales e instrumentos de medida.

CONSTRUCCIÓN: Es la etapa del hacer, el estudiante desarrolla la habilidad de representar el conocimiento a través de la construcción de un hipertexto o de la construcción de maquetas y prototipos (tecnología). En ésta los estudiantes ponen toda su creatividad en el diseño de cada una de las pantallas que conforman cada uno de los nodos de la representación previamente establecida por el equipo de trabajo.

\section{- Modelo representativo de la innovación}

El modelo representativo para llevar a cabo la innovación en el CED Venecia, es un planteamiento que busca fortalecer las competencias a través de la elaboración de estructuras de marcos (Winston, 1 992), con el propósito de formar habilidades cognítivas, metacognitivas, colaborativas y tecnológicas. La Unidad de aprendizaje (U.A.) integra los elementos del entorno en forma de escenario propicio para llevar a cabo este objetivo presentado con el carácter de innovación para la Institución.

El modelo de la Unidad de aprendizaje comprende 5 etapas que alternan el carácter individual y colaborativo según Los momentos de la innovación y que se identifican así:

- Módulo de representación individual.

- Módulo de representación Colaborativa.

- Módulo Individual de solución de problemas

- Módulo Colaborativo de elaboración de hipertextos.

- Módulo Individual de acreditación.

En la siguiente gráfica se muestra la UA y se identifican cada una de las partes que la componen. 


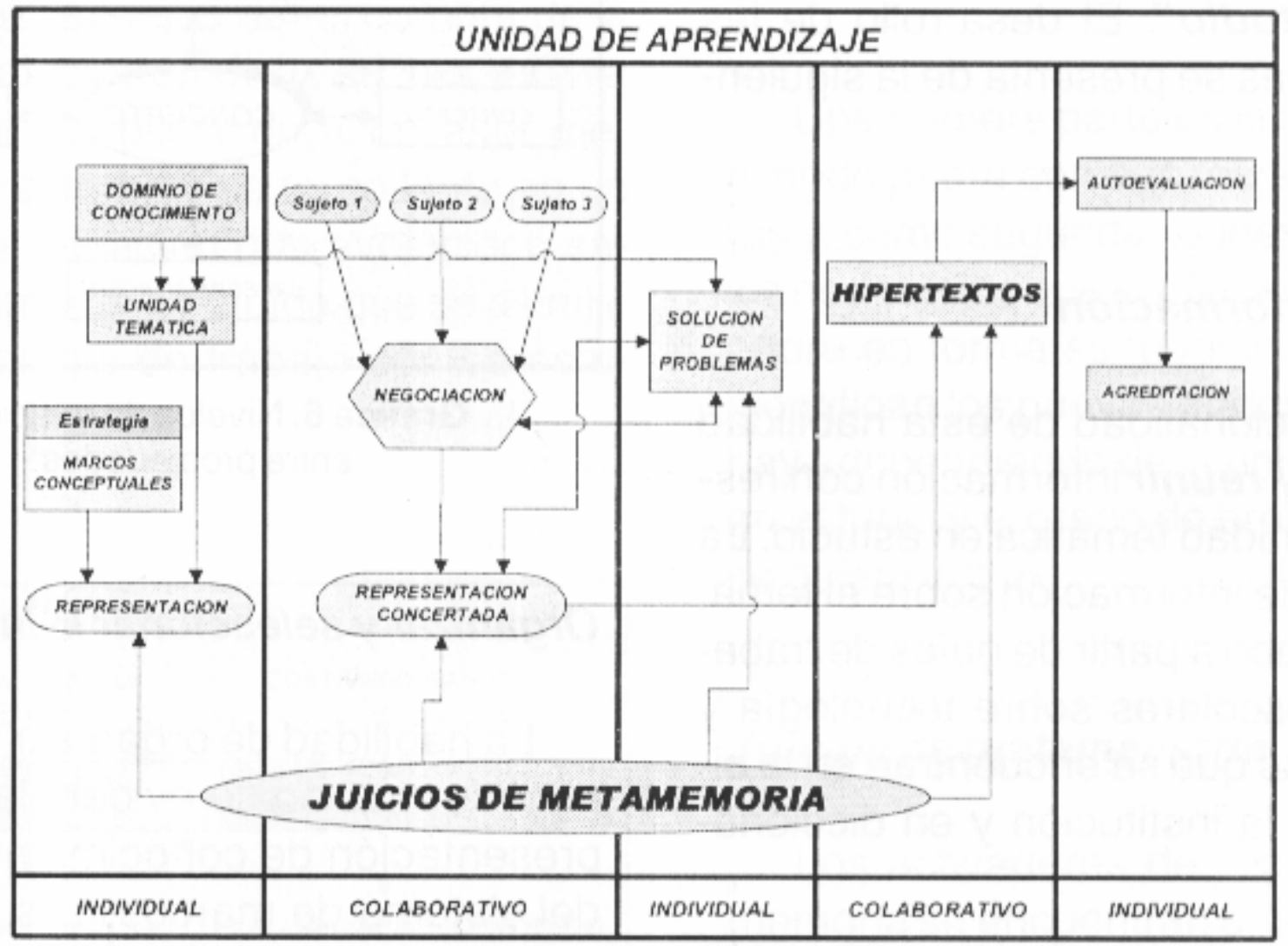

Gráfica 4. Modelo representativo de la innovación.

\section{INICIO DE LA INNOVACIÓN EDUCATIVA}

Los profesores participantes realizamos talleres de preparación, con el objetivo de construir el modelo representativo de la innovación y el marco teórico que lo sustenta. A través de la sensibilización se logró, de igual forma, unificar criterios de trabajo, metas a alcanzar, selección de unidades temáticas a tratar en cada una de las áreas, disponibilidades de tiempo y horarios de trabajo; se definió la metodología de trabajo con Los estudiantes y la forma como se implementaría el modelo de la innovación en el aula de clase y

se definieron los roles de los profesores, estudiantes y grupo asesor en torno al desarrollo de la Unidad de Aprendizaje propuesto en la innovación educativa.

\section{IMPLEMENTACIÓN DEL MODELO DE LA INNOVACIÓN EN EL AULA DE CLASE}

A continuación se mostrará la forma como de implemento el modelo de la innovación con los estudiantes y cómo se lleva cabo la metodología de trabajo, explicando cada módulo.

\section{MÓDULO 1}

\section{Representación individual}

El trabajo individual de los estudiantes busca desarrollar competencias cognitivas. Este se inicia con el planteamiento de una situación problemática en la guía de trabajo, la cual se indica en términos de: "representar por medio de un sistema de marcos la 
unidad temática en estudio". El desarrollo de las competencias se presenta de la siguiente manera:

\section{Adquirir información}

La intencionalidad de esta habilidad es buscar y reunir información con respecto a La unidad temática en estudio. La búsqueda de información sobre el tema, se lleva a cabo a partir de guías de trabajo, textos escolares sobre tecnología y matemáticas que se encuentran en la biblioteca de la institución y en diccionarios.

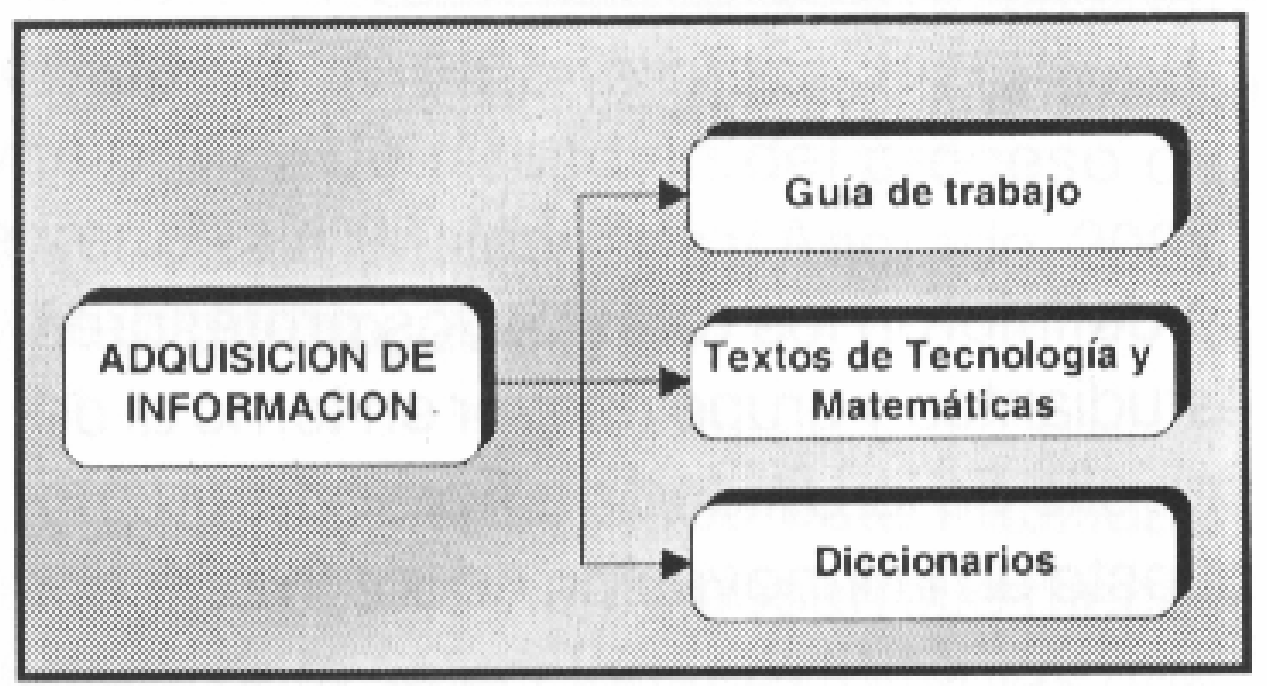

\section{Gráfica 5. Búsqueda de información.}

\section{Relaciones causa - efecto}

Esta habilidad se evidencia con las inferencias lógicas que hacen los estudiantes al relacionar definiciones con explicaciones asociadas al contexto en el cual se encuentran ubicados. En el área de tecnología, se realizaron talleres experimentales, donde el alumno confrontó Lo teórico con Lo práctico para establecer de esta manera, un número mayor de relaciones, entre definiciones y explicaciones. 


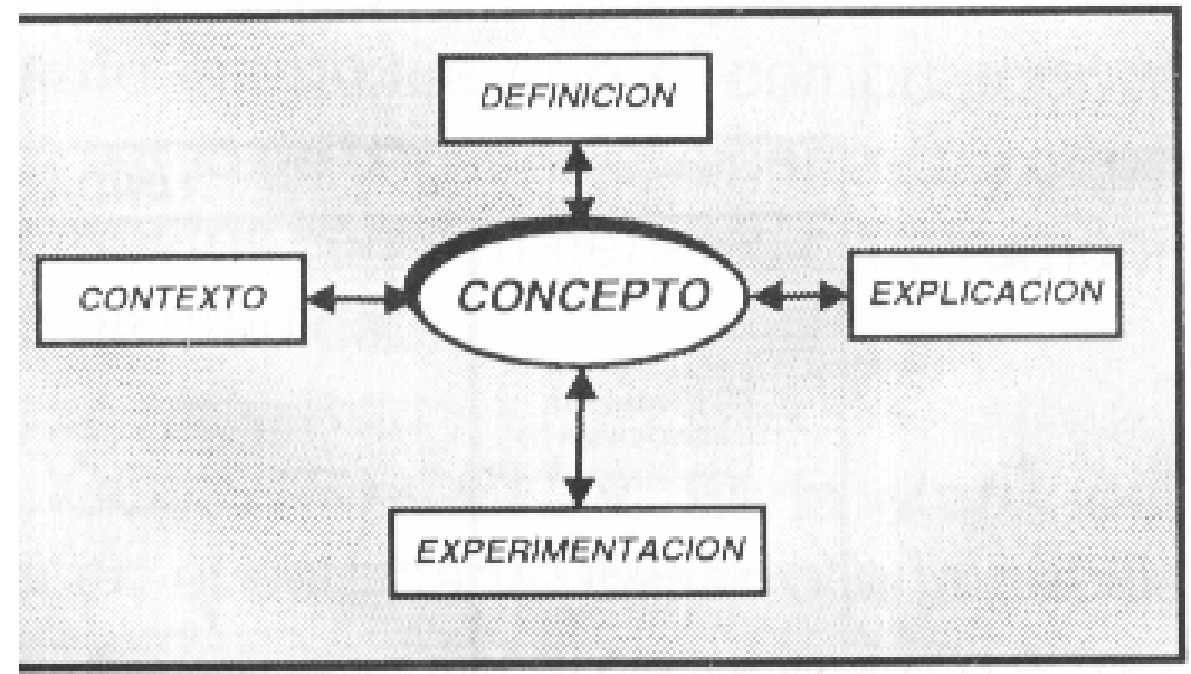

Gráfica 6. Niveles de relaciones entre propiedades.

\section{Organizar y seleccionarla información}

La habilidad de organizar y seleccionar información se evidencia con la representación de conocimiento a través del sistema de marcos. Las relaciones de herencia, entre el nodo padre y los nodos hijos, se hace con base en la información que el alumno tenga sobre Las unidades temáticas en estudio. Los alumnos, a nivel individual, trabajan el concepto de herencia con la ranura instancia.

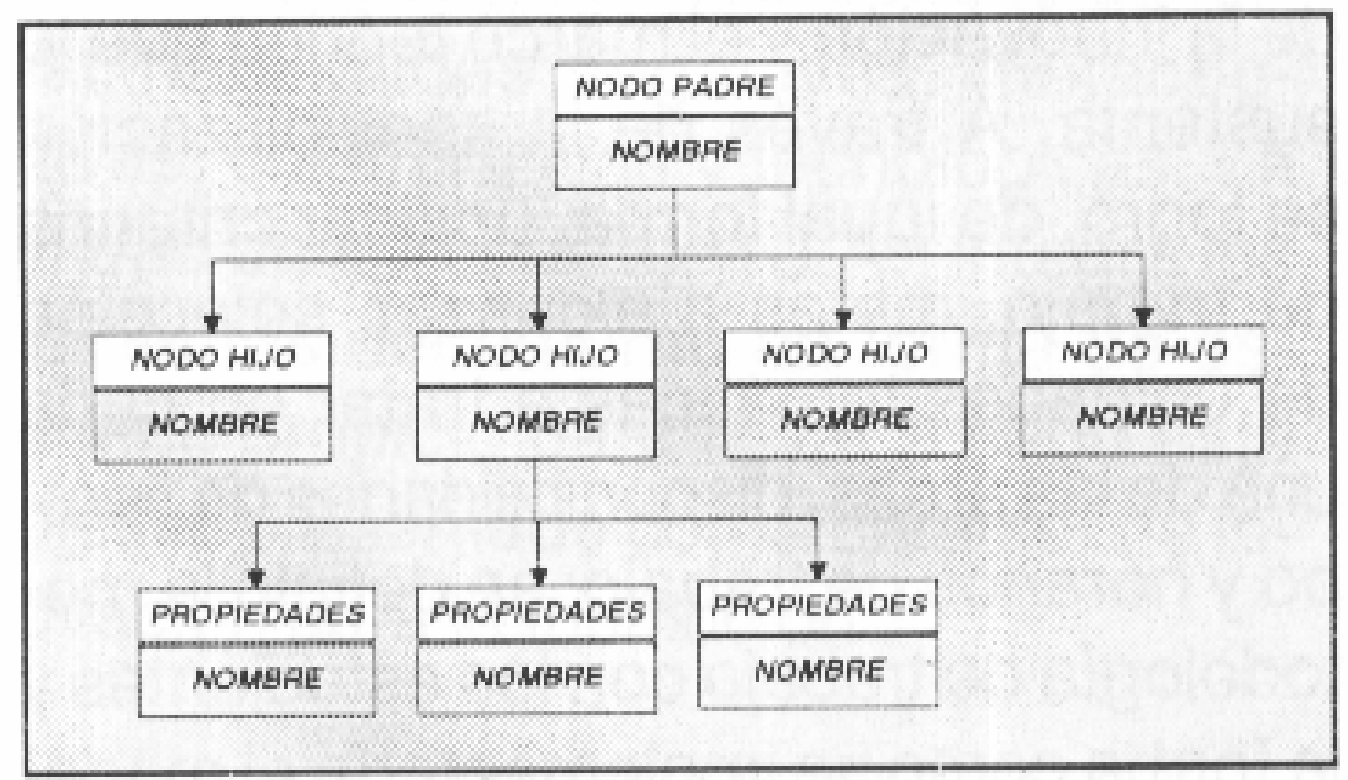

Gráfica 7. Organizar la información. 
La organización de la información obtenida por el estudiante, es procesada por medio de categorías jerárquicas, donde se identifican las instancias y se selecciona el conjunto de ranuras que conforman el nodo padre y cada uno de los nodos hijos.

Es importante en esta etapa el desarrollo del modelo de ranuras, ya que el estudiante tiene que dar el nombre de la ranura, su contenido y su respectiva definición y explicación. Con esta metodología el trabajo se tomó lento en un comienzo, situación que evolucionó satisfactoriamente a medida que se asimiló la metodología de trabajo por los estudiantes.

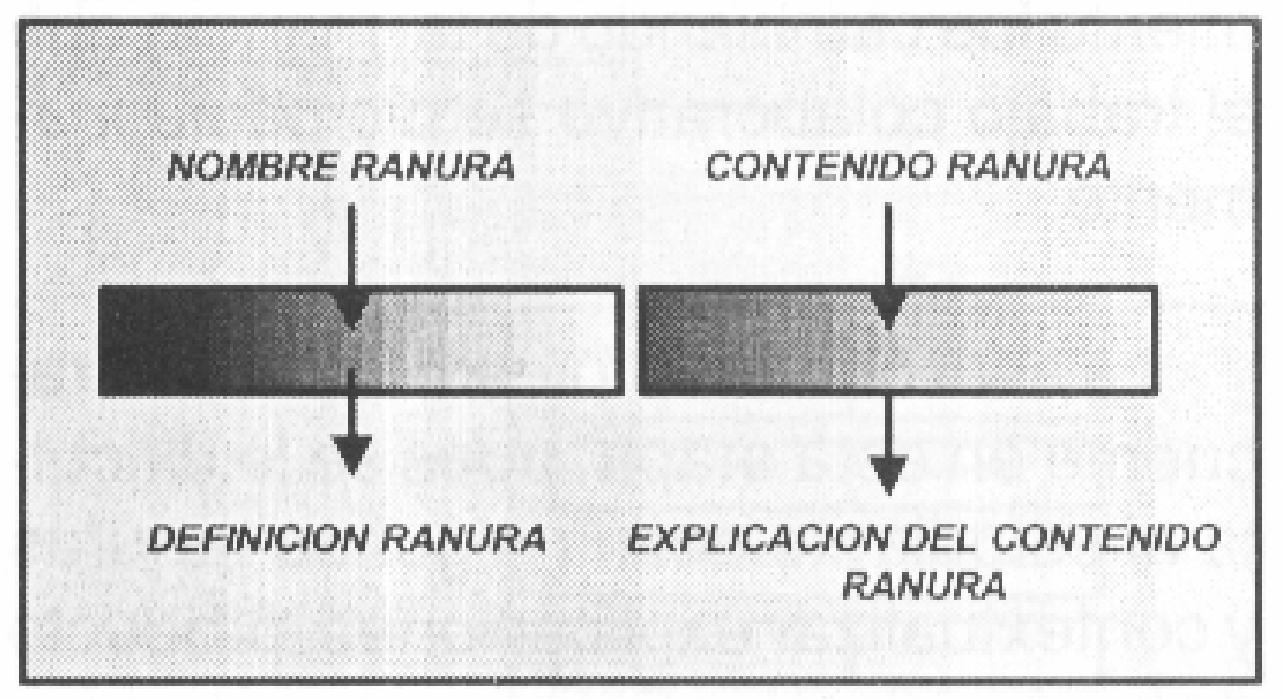

\section{Gráfica 8. Modelo de las ranuras.}

\section{Descomposición de un problema en subproblemas}

Con la información hasta ahora recolectada y seleccionada por los estudiantes, se explica la metodología de la descomposición de un problema en subproblemas. Para este caso especifico, la estructura del conocimiento a través de marcos se subdivide en varias partes.

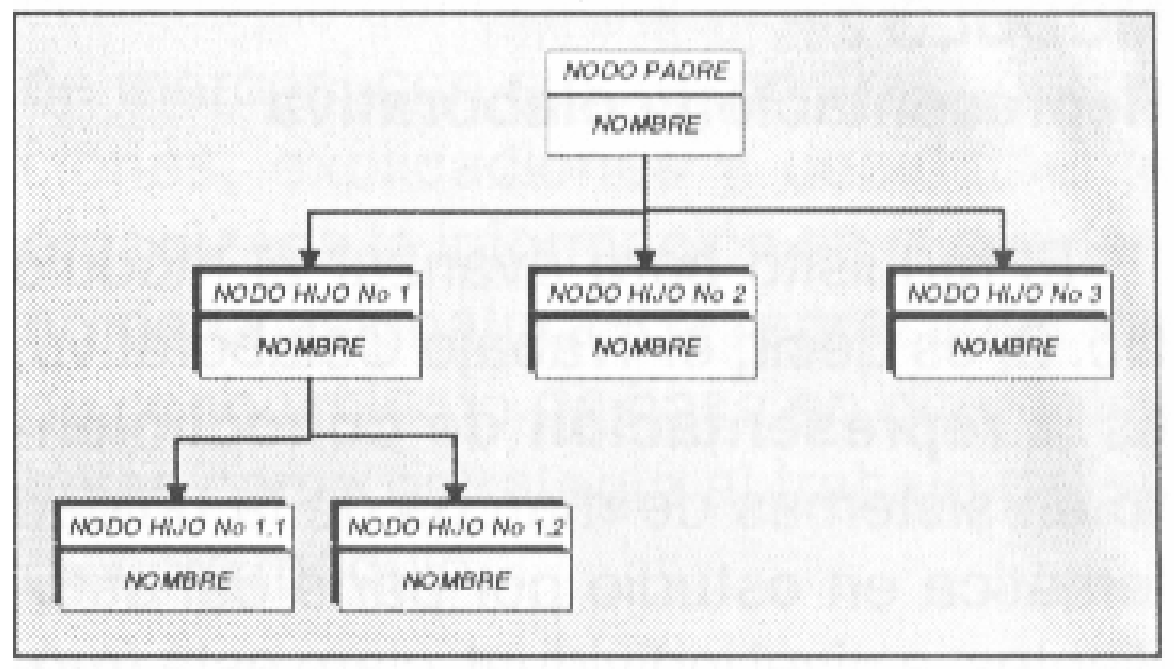

Gráfica 9. Descomposición de un problema. 
Una primera parte es la identificación el nodo padre con sus respectivas ranuras y, como segunda, la identificación de Los nodos hijos que se derivaban del nodo padre en forma secuencial. Al igual, se identifican los nuevos nudos hijos —si los haydependiendo de la unidad temática en estudio y el grado de profundidad que se quiera lograr.

\section{Juicios de metamemoria}

Los activadores de juicios de mata-memoria se encuentran el la guía de trabajo. Los estudiantes los leen y responden a cada uno de ellos. El objetivo de estos es obtener propuestas de metas por parte del alumno en relación con la solución de la guía de trabajo.

Como meta para llegar al trabajo colaborativo, es decir, módulo 2, los estudiantes, a nivel individual, tienen que hacer una representación del nodo padre, con sus respectivas ranuras, definiciones y explicaciones. La representación se hace en el cuaderno de la asignatura en forma de rectángulos, siguiendo el modelo propuesto por Winstan (1992).

\section{MÓDULO 2}

\section{Representación colaborativa}

El requisito para avanzar al módulo No. 2, es decir, el Trabajo Colaborativo, es la representación de conocimiento en sistemas de marcos de la unidad temática en estudio por parte del estudiantes, a nivel individual, claro está (módulo 1).

El trabajo Colaborativo se inicia con la conformación, por parte del profesor, de un equipo de trabajo de tres estudiantes, equipo que va a tener como meta final una representación de conocimiento concertado.

El objetivo de este módulo, es potenciar en el estudiante la competencia colaborativa, la cual se basa en el trabajo en equipo para llegar a la solución de la situación problemática planteada en un comienzo. En esta etapa cada estudiante emprende un proceso de negociación de saberes, teniendo como meta una representación de conocimiento más estructurada que la individual.

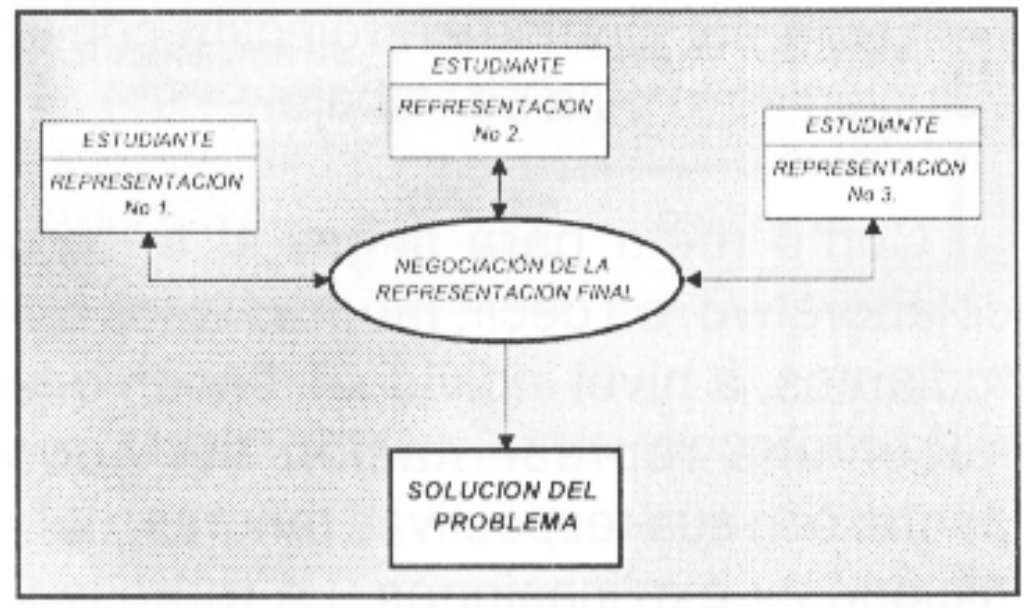

Gráfica 10. Proceso de negociación de saberes. 
En el desarrollo de este módulo, los estudiantes se reúnen en una mesa de trabajo y cada uno muestra su representación de conocimiento. En un comienza, el trabajo se torna difícil, pues los niños nunca han experimentado el trabajada en equipo, por esta razón muestran un comportamiento apático frente al proceso. No hay una participación activa de cada uno de los integrantes del equipo en torno a la negociación de sabores, en algunos equipos se presenta empatía entre los integrantes. El profesor juega un papel muy importante en esta etapa, ya que tiene que estar atento de que el proceso se dé, prestando asesoría y acompañamiento permanente a los diferentes equipos de trabajo.

En la medida en que pasa el tiempo, la receptividad de los estudiantes frente al proceso fue positiva y su comportamiento fue cambiando dotal manera que el trabajo colaborativo se dio paulatinamente.

La asesoría del profesor debe ser $f$ recuente en esta etapa, pues se le dificulta al estudiante definir algunas ranuras y contextualizar explicaciones, producto de la negociación. Este proceso demanda mayor exigencia en el nodo padre, mientras que en el desarrollo de los nados hijos, la intervención del profesor es menor.

\section{Juicios de metamemoria}

Al equipo de trabajo (unidad colaborativa), conformado para la realización de una representación de conocimiento concertada, se le hacen una serie de preguntas (juicios de metamemoria), como estrategia para activar diferentes niveles de reflexión del conocimiento, hasta ahora obtenido en el trabajo individual. Estos juicios se hacen antes de hacer dicha representación. Los estudiantes leen los juicios, los analizan y sus respuestas ya no son tan ligeras y carentes de argumentación como en el trabajo individual, pues se ha adquirido mayor experiencia con el trabajo previo a este módulo.

Como resultado final de este módulo, se obtiene una representación con concertada de conocimiento en estructura de marcos, con sus respectivas definiciones, explicaciones y debidamente organizada la información en el cuaderno de la asignatura. La representación de conocimiento de hace en diferentes hojas como estrategia al trabajo del siguiente módulo.

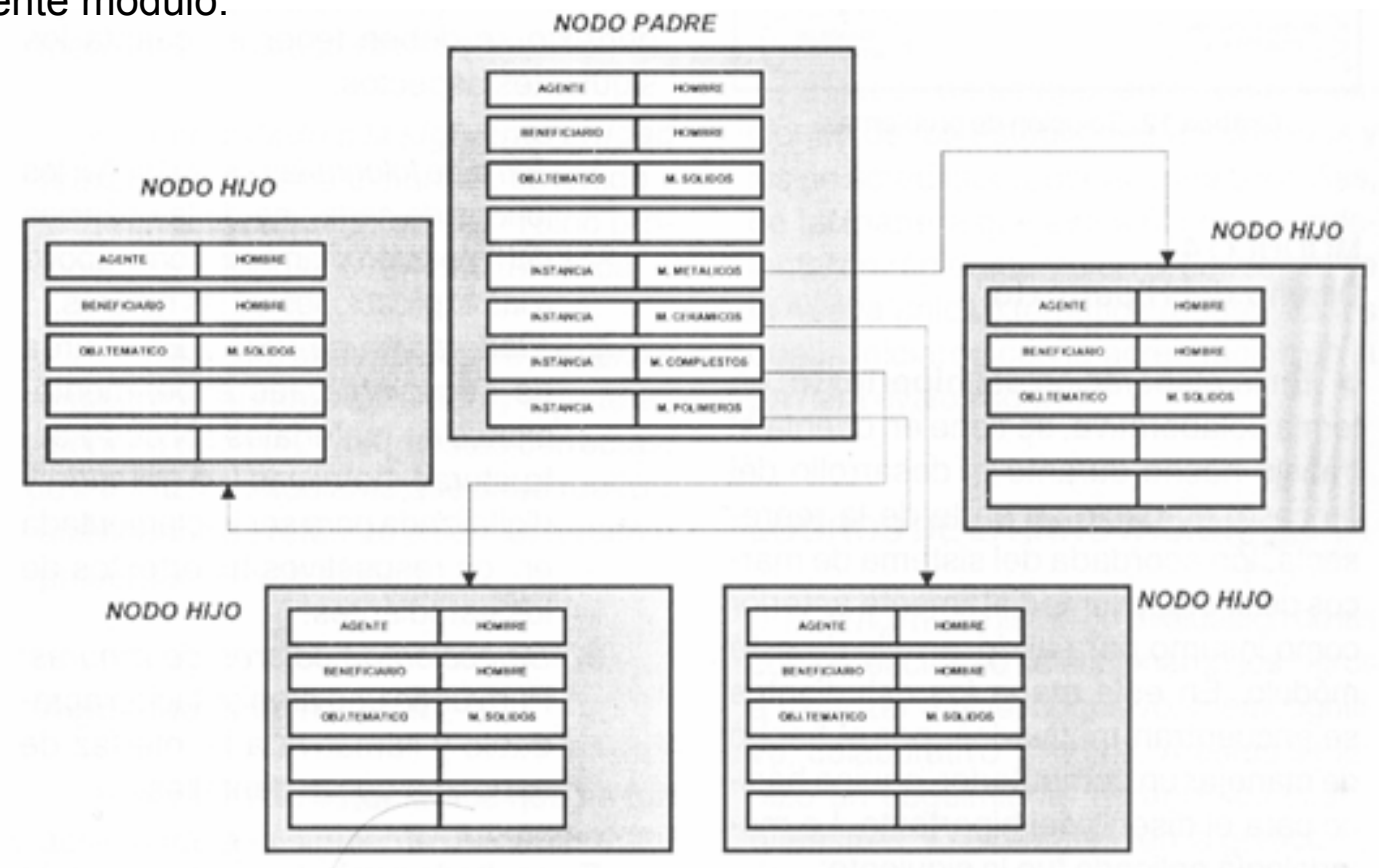

Gráfica 11. Representación concertada (Trabajo colaborativo). 


\section{MÓDULO 3}

\section{Solución de problemas (individual - colaborativa)}

En el módulo No. 3., la solución de problemas, se evidencia en el sistema de marcos de la unidad temática en estudio. La solución del problema a nivel colaborativo es más estructurada y elaborada por ser resultado de la interacción y negociación de tres estudiantes.

Para obtener la representación final en el sistema de marcos, los estudiantes, tanto a nivel individual como colaborativo, desarrollan una serie de estrategias que en su momento son decisivas para la solución del problema. Se evidenciaron estrategias fuertes en la solución de problemas en cada uno de las unidades colaborativas. Cada grupo conceptualiza y contextualiza los conocimientos aprendidos de tal forma que la representación final se ajusta a la realidad vivenciada por los estudiantes.

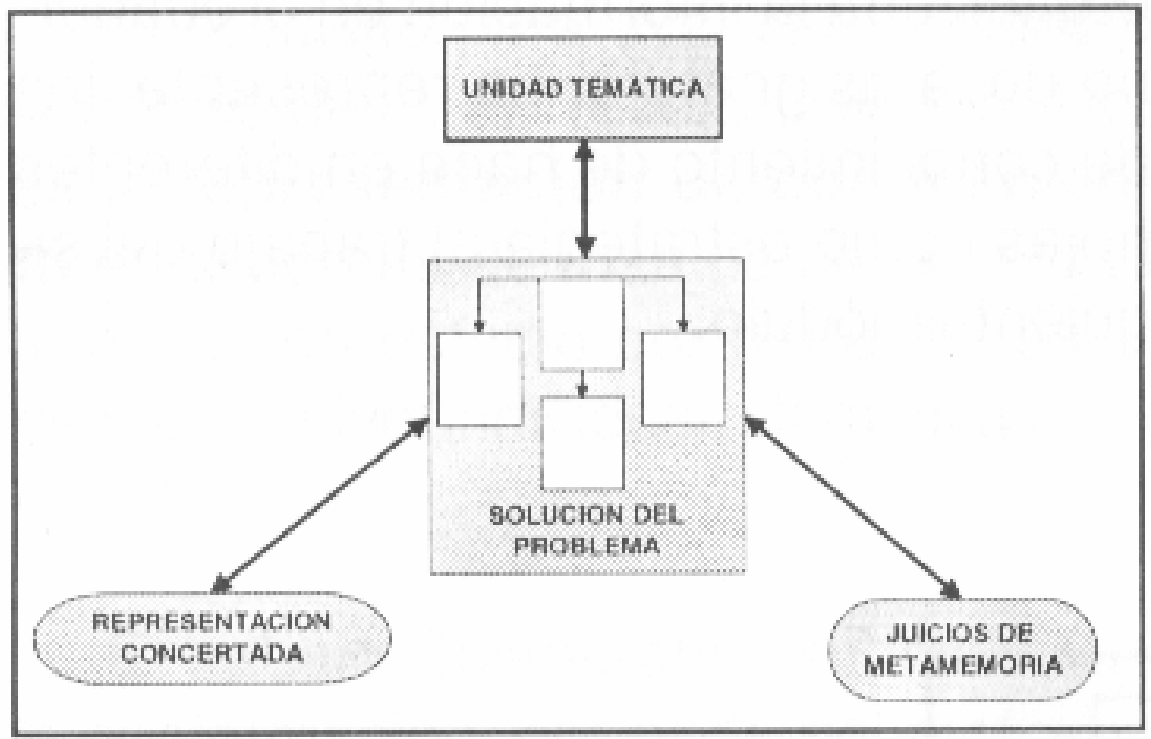

Gráfica 12. Solución de problemas.

\section{MÓDULO 4 \\ Diseño del Hipertexto}

En la elaboración del hipertexto, en forma colaborativa, se tiene en cuenta el trabajo hecho durante el desarrollo del proceso, es decir, se parte de la representación acordada del sistema de marcos de la etapa inmediatamente anterior como insumo para el desarrollo de este módulo. En esta etapa los estudiantes se encuentran motivados, por el hecho de manejar un computador, equipo básico para el diseño del hipertexto. La metodología aplicada fue la siguiente:

\section{Trabajo con el shell}

En esta etapa, se explica la forma operar el Shell (pieza de software para desarrollar hipertextos). Los estudiantes se dividieron el trabajo, de tal forma que uno de ellos digita la información contenida en el cuaderno, mientras que los otros dos le dictan y están pendientes de la correcta operación del shell. En la medida en que se avanza en la digitación de ellos, el shell hace preguntas y espera ingreso de respuestas por parte de los alumnos, metodología no asimilada correctamente por los estudiantes al comienzo, 
pero en la medida en que se interactúa con el sistema el avance en su manejo es significativo y sin contratiempos.

\section{Diseño gráfico del Hipertexto}

Para hacer el diseño gráfico del hipertexto se deben tener en cuenta los siguientes aspectos:

1. Toma de fotografías. Para diseñar los fondos de cada una de las páginas del hipertexto y también como apoyo a las explicaciones de las ranuras.

2. Elaboración de Vídeo: En el área de Tecnología, las experimentaciones de materiales sólidos y estructuras metálicas fue grabada y digitalizada para ser implementada en los respectivos hipertextos de los estudiantes.

3. Selección de colores de ranuras: Para darle una ambientación agradable y llamativa a la interfaz de cada una de las pantallas.

El resultado arrojado por la aplicación de este módulo se traduce en la elaboración del hipertexto como una pieza de software funcional.

\section{MÓDULO 5 \\ Autoevaluación}

\section{Evaluación}

El módulo de Autoevaluación, es el módulo final que cierra el modelo de la innovación. Es aquí donde el estudiante pide ser evaluado por el profesor de la asignatura. Para esto, se diseña una evaluación tendiente a establecer si el alumno posee los elementos necesarios a nivel metodológico y conceptual que le permitan ser acreditado a la siguiente unidad temática. Esta evaluación es individual. La evaluación comprende veinte (20) preguntas, de tal forma que se requiere un mínimo de doce (12) respuestas correctas para aprobar la unidad temática en estudio. Hay dos tipos de mensajes a los estudiantes:

- Acreditado a la siguiente unidad temática: Muestra el puntaje obtenido y le informa al estudiante que ha sido promovido a la siguiente unidad temática.

- Necesita actividades de refuerzo: Muestra el puntaje obtenido y si este es menor a doce (12) respuestas correctas, le informa al estudiante que debe realizar actividades de refuerzo para nuevamente presentar la evaluación.

\section{Actividades de refuerzo}

El número de estudiantes que no fue acreditado a la siguiente unidad temática no es significativo en ninguna de las asignaturas y en ninguna de las unidades temáticas en estudio, pues osciló entre el 10 y el 15\%. Para este grupo de estudiantes, se adelantan actividades de refuerzo, tales como ejercicios prácticos tanto en el área de matemáticas como de tecnología. Al ser nuevamente evaluados los estudiantes, si llenan los requisitos, serán promovidos a la siguiente unidad temática.

El lapso de tiempo, entre las evaluaciones de los estudiantes acreditados y los no acreditados, osciló entre 3 y 4 días, de tal manera que estos últimos se adelantaron 
rápidamente en el comienzo de la nueva unidad temática respecto a los que la iniciaron con la acreditación en la primera evaluación.

\section{LOGROS DE LA INNOVACION}

Como mecanismo de evaluación de las competencias se seleccionaron los logros a alcanzar a nivel cognitivo, metacognitivo, colaborativo y motriz. A éstos se les hizo un seguimiento durante los cuatro bimestres, y se resumen en la gráfica 13.

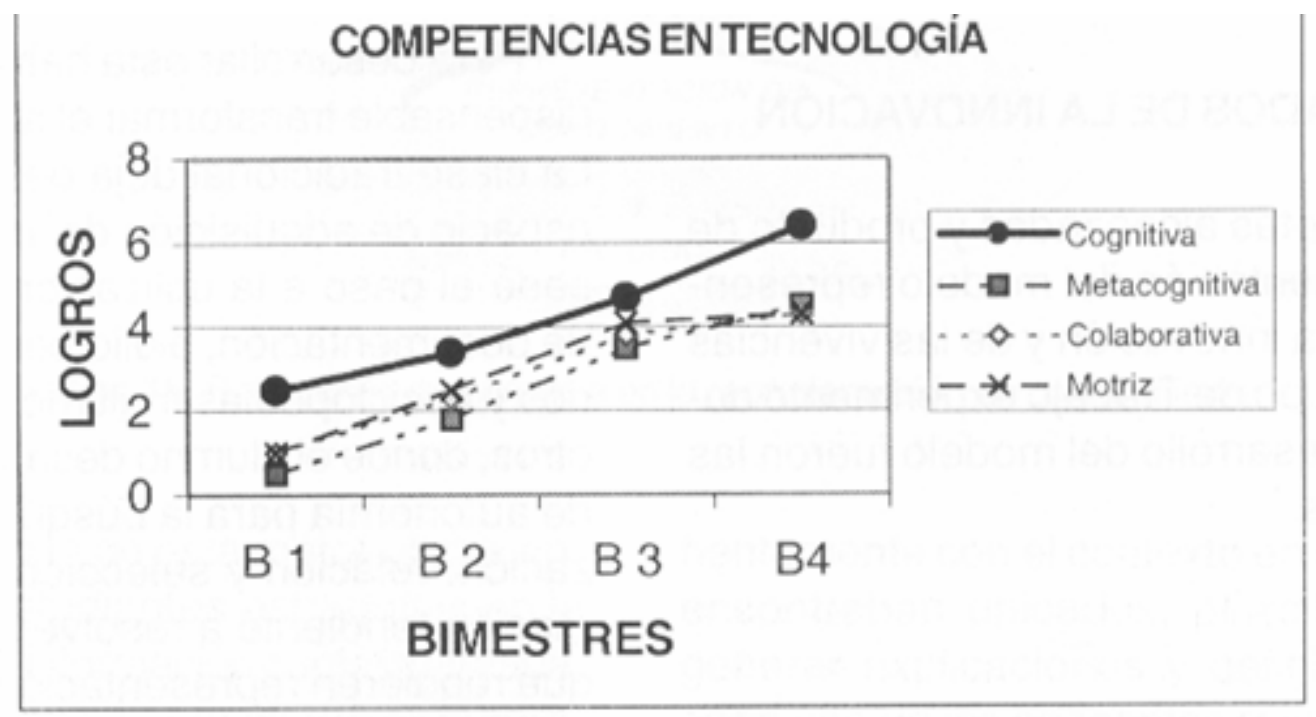

Gráfica 13. Logros en tecnología.

De acuerdo con la gráfica 13, que representa el promedio de logros alcanzados por los estudiantes en el área de tecnología se observa un incremento progresivo a través de los períodos académicos.

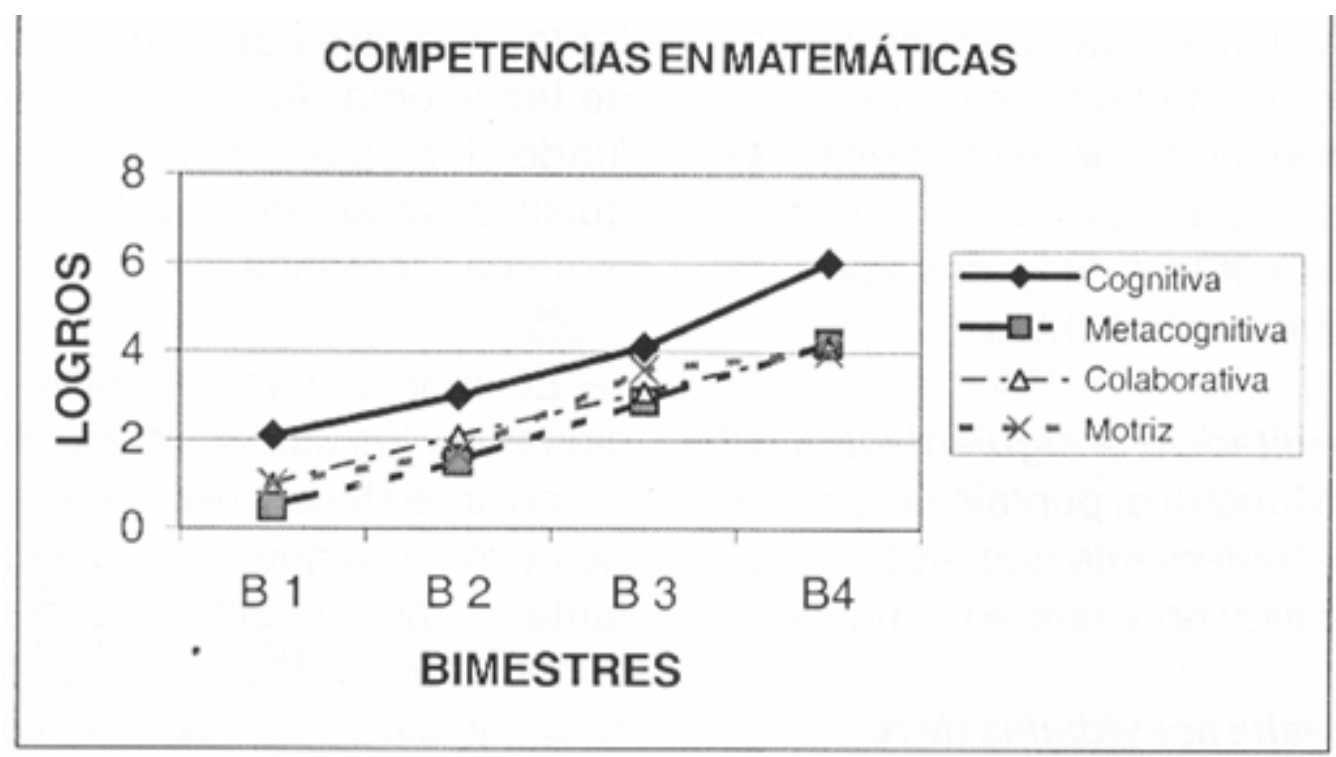

Gráfica 14. Logros en matemáticas. 
En el área de las matemáticas encontramos un comportamiento similar, aunque no tan acentuado como en el área de tecnología. Al finalizar el cuarto bimestre todos los alumnos alcanzan los logros propuestos, sin embargo algunos estudiantes necesitaron actividades de refuerzo.

\section{RESULTADOS DE LA INNOVACIÓN}

Las metas alcanzadas y producto de la implementación del modelo representativo de la innovación y de las vivencias que el Grupo de Trabajo experimentó durante el desarrollo del modelo fueron las siguientes.

\section{- Habilidad cognitiva}

El desarrollo, en los estudiantes, de esta competencia genera habilidades mentales que les van a servir para afrontar situaciones problemáticas en la solución de problemas en cuanto a la representación de conocimiento.

Para desarrollar esta habilidad fue indispensable transformar el aula de clase. La clase tradicional deja de ser el único espacio de adquisición de información y sede el paso a la utilización de centros de documentación, bibliotecas, laboratorios y enciclopedias multimediales, entre otros, donde el alumno desarrolla niveles de autonomía para la búsqueda, organización, relación y selección de la información tendiente a resolver problemas, que requieren representación de conocimiento para su solución.

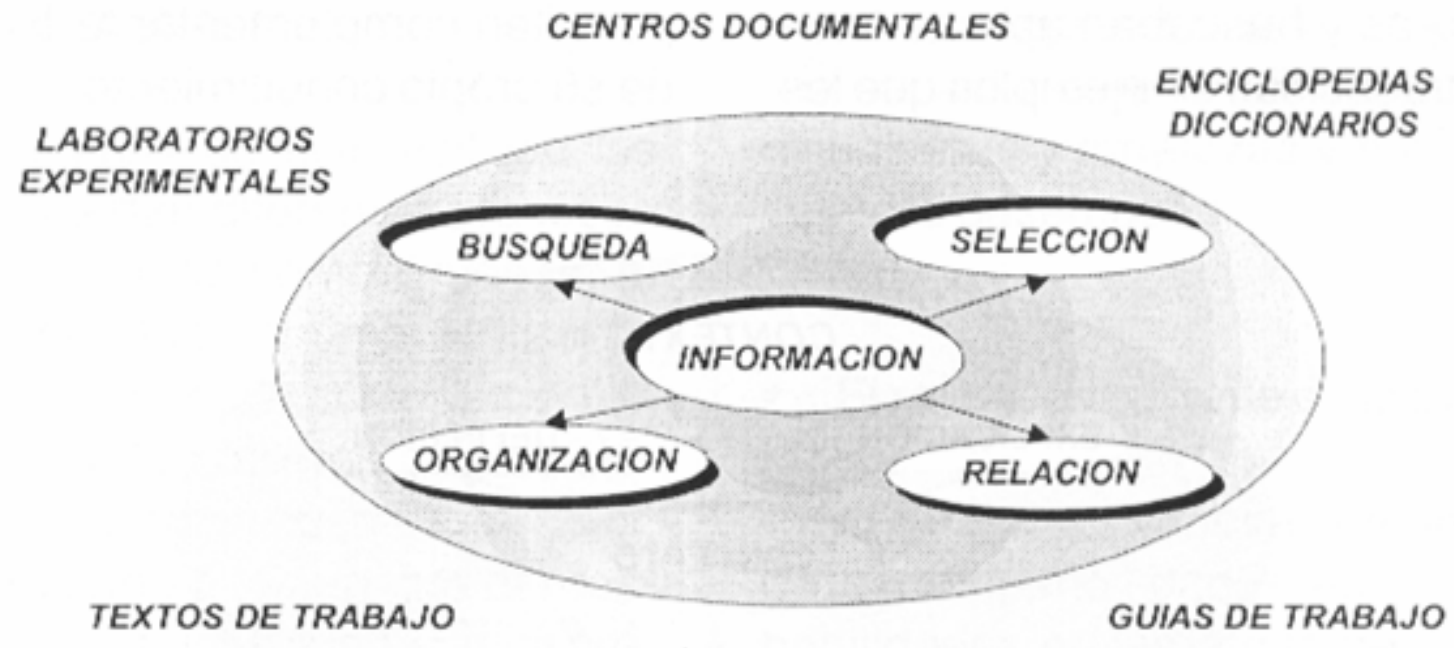

BIBLIOTECAS

Gráfica 15. Búsqueda de información. 
De igual forma con la habilidad de subdividir un problema en subproblemas, se constituyó en una estrategia fuerte en la solución de los mismos. El estudiante posee más elementos de juicio para estructurar de una forma holística la información y la especificidad a la que se quiere llegar en cada una de las temáticas abordadas.

La representación de conocimiento a través del sistema de marcos, se convirtió en el escenario propicio para desarrollar las habilidades de adquisición de información, organización y selección de la información, descomposición de un sistema en subproblemas y la forma en que el estudiante relaciona la información que posee en su memoria de largo plazo con la información que tiene en su memoria de corto plazo cuando se enfrenta a la solución de problemas de representación de conocimiento.

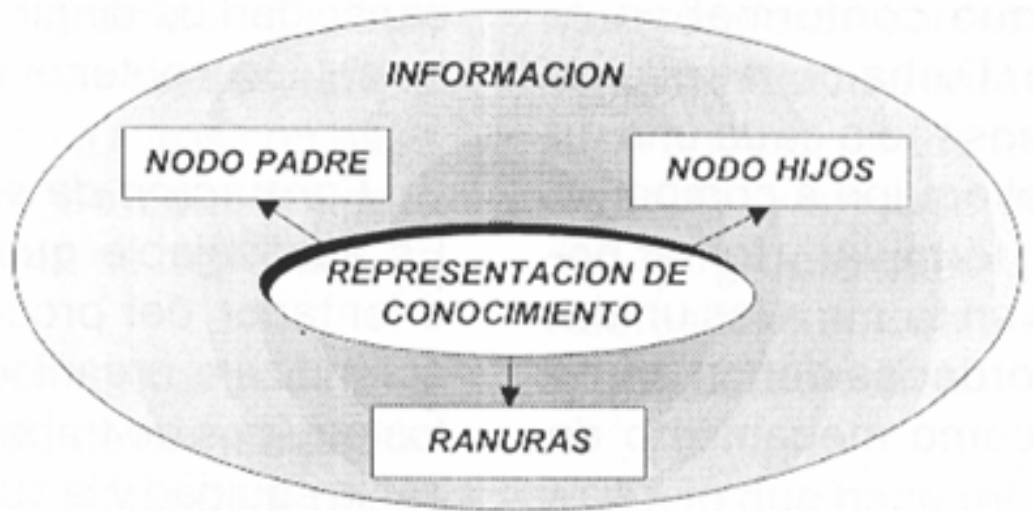

\section{Gráfica 16. Relaciones de herencia en la representación de conocimiento.}

El desarrollo de esta metodología enseñó a los estudiantes estrategias en la búsqueda de información contextualizada, pues la información era asociada permanentemente con el contexto en el cual se encontraban ubicados, permitiéndoles generar explicaciones y definiciones a cada uno de los conceptos relacionados en las ranuras y buscaban aplicaciones concretas traducidas en ejemplos que les permiten complementar la construcción de su propio conocimiento.

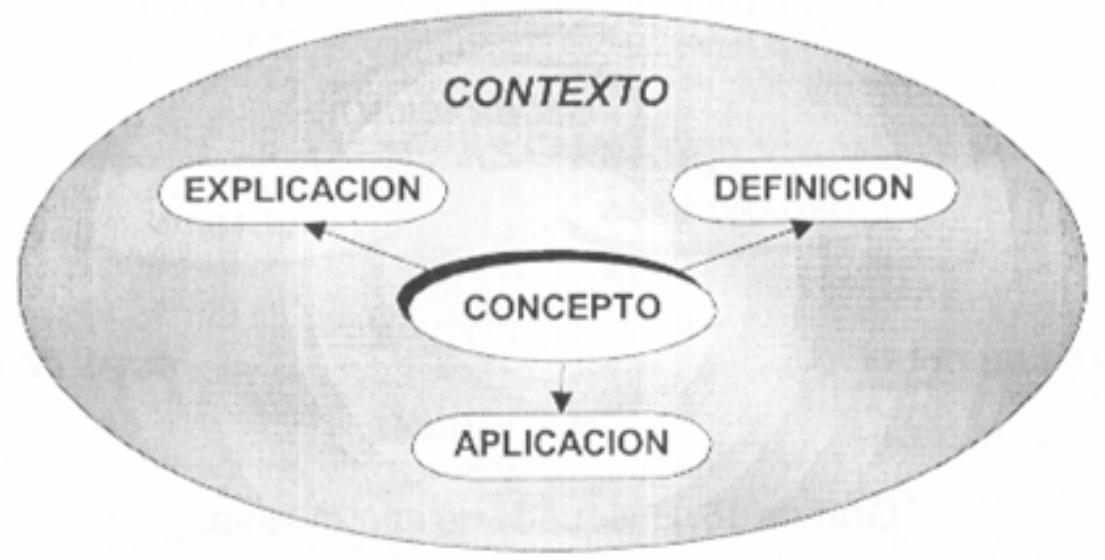

Gráfica 17. Contextualización de la información. 


\section{- Habilidad colaborativa}

El desarrollo de esta habilidad adquirió aplicación en Los siguientes campos:

Formación académica: La negociación de saberes en torno a la solución de problemas de representación de conocimiento se constituyó en un espacio de interpretación y argumentación de cada una de las representaciones de los estudiantes que conformaban el equipo de trabajo. Dicha construcción de conocimiento enseñó a cada uno de los integrantes del equipo a compartir, debatir, sustentar, formular y tomar posiciones objetivas en tormo a las unidades temáticas abordadas donde primó el conocimiento como mecanismo de negociación.

Formación de valores: El trabajo colaborativo permite a los estudiantes interactuar con los demás miembros del equipo de trabajo y de esta forma, se constituye en un espacio de formación del individuo en valores, tales como, tolerancia, respeto, disciplina, solidaridad y de conciliación de sabe res. Al despertar en los alumnos, valores de esta clase, estamos creando las verdaderas escuelas de paz y convivencia.

Formación de líderes: El trabajo colaborativo permite identificar y potenciar el espíritu de liderazgo entre los miembros del equipo. La formación de líderes es un reto que debe asumir el docente para potenciar en el estudiante la capacidad de dirigir y orientar el trabajo en equipo y obtener mejores resultados.

Formación de equipos de trabajo: Es aconsejable que el profesor, como orientador del proceso de enseñanza aprendizaje, organice la conformación de los equipos de trabajo, tratando de buscar la equidad y la cualificación académica. Como segunda instancia, se debe evitar que siempre trabajen los mismos integrantes del grupo de trabajo, deben ser rotados, de tal manera que se de la oportunidad de interactuar con el mayor número posible de compañeros para fortalecer Los lazos de fraternidad y de amistad de los integrantes del curso.

\section{- Habilidad motriz}

Con el desarrollo de esta habilidad, los alumnos despiertan diferentes procesos motrices, tanto finas como gruesas, al manipular diferentes instrumentos tecnológicos (computador). La construcción de un prototipo, en los talleres de tecnología, se constituyó en un agente motivador, pues los estudiantes tenían que dar respuesta a una necesidad y fabricar un prototipo producto de un diseño concebido inicialmente. Es básico que el estudiante combine la parte teórica con la práctica a través de los talleres experimentales para confrontar conceptos y generar su propio conocimiento como actividad complementaria a la representación de conocimiento a través del sistema de marcos.

El diseño de un hipertexto nace de una representación de conocimiento consignada en forma escrita, la cual se concretiza en un producto final (software), con base en una planeación y organización de una serie de actividades que conllevan a alcanzar esta meta. De esta forma, los alumnos, que vivenciaron el proceso de diseño de piezas de software, desarrollaron estrategias fuertes para la solución de problemas futuros con base en representaciones de conocimiento estructuradas — sistema de marcos-.

El desarrollo de la habilidad motriz se constituye en el puente de comunicación entre lo teórico y lo práctico para poder ejecutar y plasmar en una realidad lo concebido teóricamente. 


\section{- Habilidad metacognitiva}

El desarrollo de la habilidad metacognitiva en los estudiantes favorece la autonomía en el aprendizaje, la autovaloración, y la capacidad de identificar sus propias dificultades y fortalezas y mejora los niveles de desempeño.

\section{- El rol del estudiante en la innovación}

Al final de la innovación, los niveles de desempeño, dados en términos de habilidades en las áreas de dominio de conocimiento (Matemáticas y Tecnología), se evidencian en la validación del diseño del hipertexto y en la evaluación individual hecha a cada uno de los estudiantes al final de cada unidad temática.

El rol del estudiante dejó de ser pasivo para convertirse en dinámico, pues en éste, él tiene que buscar la información que se encuentra en diferentes fuentes, seleccionar la que más se ajuste a la resolución del problema, contando con el profesor, durante el desarrollo de la actividad, como un orientador del proceso.

Al hablar de habilidades colaborativas, es indispensable mencionar que los alumnos rompieron con el esquema de sólo estudio individual para integrarlo al trabajo en equipo, donde tenían que planear, organizar y ejecutar diferentes actividades en grupo, generando una serie de valores frente al esfuerzo individual y al aporte del colectivo.

En lo que hace referencia a la lectoescritura y redacción, el papel de los estudiantes cambió significativamente, pues de ser el alumno que copiaba la información que el profesor le suministraba en el aula de clase y que repetía al pie de la letra, pasó a ser la persona que redacta sus propias definiciones y explicaciones con base en la búsqueda de información de la unidad temática en estudio.

\section{- El rol del profesor}

El profesor fue un facilitador del proceso enseñanza- aprendizaje frente a los estudiantes. Su papel primordial fue:

1. Observador de procesos: Monitoreaba el desarrollo de las actividades y el nivel de aprendizaje del alumno.

\section{Diseñador de guías de trabajo:}

El profesor decidió la estructura y contenidos de las unidades temáticas, de tal forma que respondieran a necesidades pedagógicas que la innovación debía satisfacer.

3. Orientador de procesos: Fue un guía en el proceso de aprendizaje de los estudiantes. Su papel estuvo encaminado a facilitar los mecanismos y metodologías necesarias para que los alumnos alcanzaran los niveles de competencias requeridos.

\section{Incorporando las TIC en el aula}

de clase: Este tipo de innovación pedagógica exigió que el docente se cualificara y utilizara las tecnologías de la información y de la comunicación "TIC", con una concepción pedagógica. 
5. Evaluador de procesos: La formación de competencias en los estudiantes exigió que la evaluación fuera un proceso acumulativo y que se orientara, constantemente, mediante el mecanismo de retroalimentación el alumno, de tal forma que éste identificara sus fortalezas y debilidades, para lograr que éstas últimas pudieran ser superadas mediante actividades de refuerzo.

\section{BIBLIOGRAFÍA}

BJORK, ROBERT (1994). Memory and Metamemory Considerations in the Training of Human Beings. In METCALFE, Jane and SHIMAMURA, Arthur P. (Eds.). Metacognition. Cambridge, MA: The MIT Press. Preface.

DERRY, S. J.; and MURPHY, D. A. (1986). Designing Systems that Train Learning Ability: from Theory to Practice. Review of Educational Research, 56(1), 1-39.

FLAVELL, J. H. \& WELLMAN, H. M. (1977). Metamemory. In KAIL, R. V. \& HAGEN, J.W. (Eds.). Perspectives en the Development of Memory and Cognition. Hillsdale. NJ: Erbaum.

GAGNÉ, R. M. (1985). The Conditions of Learning and a Theory of Instruction. New York, N.Y.: Holt, Rinehart and Winston (fourth edition).

GARNER, Howard. (1994). Estructuras de la mente. La teoría de las inteligencias Múltiples. Editorial, México, Fondo de Cultura Económica.

GOEL, V. \& PIROLLI, P. (1992). Structure of Design Problem Spaces. Cognitive Science, Vol 16, No 3, Jul.- Sep. pp. 395 - 429.

GOONATILAKE, S (1984). Aborted Discovery: Science and Creativity in the Third World. Zed Books, Londres.

JONASSEN, D. H. (1986). Hypertext Principles for Text and Courseware Design. Educational Psychologist. 21 (4), 269 - 292.

MALDONADO G., L. F. (1989). The Effect en Performance and Learner-Sequencing Decisiors of Instructional Curriculum Maps ir a Hypertext Environment. Doctoral Dissertation, Florida State Unversity. Dissertation Abstracts international.

Maldonado G., L.F. (2001). Razonamiento Espacial y Aprendizaje Significativo: Profesores y Alumnos frente a los Juegos de Descubrimiento Basados en Computador. Bogotá: Universidad Pedagógica Nacional y COLCIENCIAS.

Ortega del Castillo, N., Maldonado G., L.F., Macías M., D. E Ibáñez, I., J. (2001). Construyendo la Autonomía en el Aprendizaje de ja Matemática. Bogotá, Universidad Pedagógica Nacional e Instituto para la Investigación y el Desarrollo Pedagógico.

PERKINS, D.N. (1986). Conocimiento como Diseño. Pontificia Universidad Javeriana. Bogotá.

SOWA, J. F. (1986). Concentual Structures: Information Processing in machine. Reading MA: Addison Wesley Publishing Company. 
SOWA, J. F. (1987). Semantics Networks. In Encyclopedia of Artificial Intelligence. New York, N.Y.: Edit. John Wiley and Sons. Edited by Stuart C. Shapiro.

Winston, P.H. (1992). Artificial Intelligence. (Third Edition). Reading, Massachusetts: Addison - Wesley Publishing. 